TRANSACTIONS OF THE

AMERICAN MATHEMATICAL SOCIETY

Volume 361, Number 10, October 2009, Pages 5417-5434

S 0002-9947(09)04665-0

Article electronically published on May 12, 2009

\title{
ANALYTIC GROUPS AND PUSHING SMALL SETS APART
}

\author{
JAN VAN MILL
}

\begin{abstract}
We say that a space $X$ has the separation property provided that if $A$ and $B$ are subsets of $X$ with $A$ countable and $B$ first category, then there is a homeomorphism $f: X \rightarrow X$ such that $f(A) \cap B=\emptyset$. We prove that a Borel space with this property is Polish. Our main result is that if the homeomorphisms needed in the separation property for the space $X$ come from the homeomorphisms given by an action of an analytic group, then $X$ is Polish. Several examples are also presented.
\end{abstract}

\section{INTRODUCTION}

All spaces under discussion are separable and metrizable. By an action of a topological group on a space we will always mean a continuous action.

Let $G$ be a Polish group acting transitively on a second category space $X$. It is not difficult to prove the following: if $A$ and $B$ are subsets of $X$ with $A$ countable and $B$ first category, then there is an element $g \in G$ such that $g A \cap B=\emptyset$ (Proposition 3.4). In other words, the group $G$ pushes countable sets apart from first category sets. The standard proof of this is based on the fact that $G$ satisfies the Baire Category Theorem. There are simple examples that demonstrate that a Polish group does not necessarily push arbitrary first category sets apart; hence this result is 'best possible'.

Let $X$ be a space. We say that $X$ has the separation property, abbreviated SP, provided that if $A$ and $B$ are subsets of $X$ with $A$ countable and $B$ first category, then there is a homeomorphism $f: X \rightarrow X$ such that $f(A) \cap B=\emptyset$. We will prove among other things that every locally compact homogeneous space has the SP and that this result cannot be generalized to Polish spaces.

This notion is related to the well-known notion of countable dense homogeneity. Recall that a space $X$ is countable dense homogeneous $(\mathrm{CDH})$ if given any two countable dense subsets $D$ and $E$ of $X$ there is a homeomorphism $f: X \rightarrow X$ such that $f(D)=E$. It is trivial to show that if $X$ is a CDH Baire space, then it has the $\mathrm{SP}$. Indeed, let $A$ and $B$ be subsets of the $\mathrm{CDH}$-Baire space $X$ with $A$ countable and $B$ first category. We may assume that $A$ is dense. Since $X$ is Baire, there is a countable dense subset $C$ of $X$ with $C \cap B=\emptyset$. Now let $f: X \rightarrow X$ be a homeomorphism such that $f(A)=C$. Thus $f$ pushes $A$ and $B$ apart. We will present in $\$ 3$ an example of a compact space which has the SP, but which is not $\mathrm{CDH}$. This shows that within the class of Baire spaces, the separation property is strictly weaker than the property of being countable dense homogeneous.

Received by the editors May 29, 2007 and, in revised form, October 18, 2007.

2000 Mathematics Subject Classification. Primary 54H05, 54E52, 03 E15.

Key words and phrases. Analytic group, meager set, countable set, Polish space.

(c)2009 American Mathematical Society Reverts to public domain 28 years from publication 
Inspired by Hrušák and Zamora Avilés [13, we prove the following:

Theorem 1.1. If $X$ is a Borel space with the SP, then $X$ is Polish.

For analytic spaces, the theorem is not true. The example constructed by Hrušák and Zamora Avilés [13] under $\mathrm{MA}+\neg \mathrm{CH}+\omega_{1}=\omega_{1}^{L}$ in their Theorem 2.6 is an analytic space $Y$ with the SP which is not Polish.

We will show however that if the homeomorphisms needed in the separation property come from an action of an analytic group, then the 'underlying' space is Polish.

Theorem 1.2. Let $G$ be an analytic group acting on a space $X$. Assume that for all subsets $A$ and $B$ of $X$ with $A$ countable and $B$ first category, there is an element $g \in G$ such that $g A \cap B=\emptyset$. Then $X$ is Polish.

Observe that $G$ may be first category; hence the proof is different from the standard proof for Polish groups that we talked about earlier.

Hence the space $Y$ constructed by Hrušák and Zamora Avilés has enough homeomorphisms for the separation property, but no analytic group acting on $Y$ achieves this. This would not be an interesting observation if there were no analytic group acting on $Y$. We will show that $Y$ admits a transitive action by an analytic group $G$ which consequently pushes points apart, but not much more since our results show that no analytic group acting on $Y$ is so rich that it pushes arbitrary countable sets apart from arbitrary fist category sets. By applying the proof of Theorem 1.2 to the space $Y$, we can push this even further. We will show that if $G$ is an analytic group acting on $Y$, then there is a countable subset $D$ of $Y$ which cannot be pushed off itself by $G$, i.e., $g D \cap D \neq \emptyset$ for every $g \in D$. We summarize this in:

Theorem $1.3\left(\mathrm{MA}+\neg \mathrm{CH}+\omega_{1}=\omega_{1}^{L}\right)$. There is an analytic space $Y$ which is not Polish but has the following properties:

(1) $Y$ has the SP.

(2) There is an analytic group acting transitively and micro-transitively on $Y$ (hence $Y$ is a coset space of an analytic group and is homogeneous).

(3) For any analytic group $G$ acting on $Y$, there is a countable subset $D$ of $Y$ such that $g D \cap D \neq \emptyset$ for every $g \in G$.

\section{TERminology}

Recall our convention that all spaces under discussion are separable and metrizable.

A space is Polish if it is completely metrizable. We call a space Borel if it is a Borel subset of some Polish space. It is well-known that if a space is Borel, then it is a Borel subset of any space it is a subspace of. An analytic space is a space that is a continuous image of a Polish space. A space is co-analytic if it is the complement of an analytic subset of some Polish space. It is well-known that a space is Borel if and only if it is both analytic and co-analytic. This is due to Souslin; see e.g., 15, 14.11]. We will also need the well-known fact that an uncountable analytic space contains a Cantor set. This is also due to Souslin, see e.g., [15, 14.13]. We adopt the terminology of Kechris [15]. That is, $\boldsymbol{\Sigma}_{\mathbf{1}}^{1}(X)$ and $\boldsymbol{\Pi}_{\mathbf{1}}^{1}(X)$ denote the collections of analytic and co-analytic subsets of $X$, respectively. Analytic sets have the following well-known closure properties. 
Proposition 2.1 ([15, 14.4]). Let $X$ and $Y$ be Polish and $f: X \rightarrow Y$ continuous. Then

(1) $\boldsymbol{\Sigma}_{\mathbf{1}}^{1}(X)$ is closed under countable unions and countable intersections.

(2) If $A \in \boldsymbol{\Sigma}_{\mathbf{1}}^{1}(X)$, then $f(A) \in \boldsymbol{\Sigma}_{\mathbf{1}}^{1}(Y)$.

(3) If $B \in \Sigma_{\mathbf{1}}^{1}(Y)$, then $f^{-1}(B) \in \Sigma_{\mathbf{1}}^{1}(X)$.

A subset $A$ of a space $X$ is called meager or first category (in $X$ ) if it is a countable union of nowhere dense subsets of $X$. If $X$ is not meager (in itself), then it is said to be of the second category. We denote the collection of first category subsets of a space $X$ by $\operatorname{MGR}(X)$. Observe that $\operatorname{MGR}(X)$ is a $\sigma$-ideal, that is, an ideal of subsets of $X$ closed under countable unions.

A space $X$ is Baire if the intersection of countably many dense open subsets of $X$ is dense, or, equivalently, if the union of any countable subfamily of $\operatorname{MGR}(X)$ has empty interior. If $X$ is not a Baire space, then by a result of Hurewicz [14] (see also [19, 1.9.13]), $X$ contains a closed copy of the space of rational numbers $\mathbb{Q}$. The following result was proved in a more general context by Levi [16] (see also [19, Exercise A.13.4]). We will use it in a similar way as in Hrušák and Zamora Avilés [13].

Lemma 2.2. Every analytic Baire space has a dense Polish subspace.

A space $X$ is completely Baire if every closed subspace of it is Baire. So by Hurewicz's result just quoted, a space is completely Baire if and only if it does not contain a closed copy of $\mathbb{Q}$. Among co-analytic spaces, this characterizes completeness, as the following interesting result shows ([15, Corollary 21.21]).

Lemma 2.3. Let $X$ be co-analytic. Then $X$ is Polish iff $X$ contains no closed copy of $\mathbb{Q}$ iff $X$ is completely Baire.

A space $X$ is called homogeneous if for all $x, y \in X$ there is a homeomorphism $f: X \rightarrow X$ with $f(x)=y$. A homogeneous space that is not first category (in itself) is a Baire space. This can be seen as follows. If it is not a Baire space, then it contains a first category nonempty open subset, say $U$. By homogeneity, $X$ has an open cover by first category open subsets. But this cover has a countable subcover. Hence $X$ is first category (in itself), which is contradictory to our assumptions. We will also need the simple fact that every clopen (= open and closed) subspace of a zero-dimensional homogeneous space is homogeneous. A space $X$ is strongly locally homogeneous (abbreviated SLH) if it has an open base $\mathcal{B}$ such that for all $B \in \mathcal{B}$ and $x, y \in B$ there is a homeomorphism $f: X \rightarrow X$ which is supported on $B$ (that is, $f$ is the identity outside $B$ ) and moves $x$ to $y$. Observe that every zero-dimensional homogenous space is SLH (the clopen sets do the job).

Let $a: G \times X \rightarrow X$ be an action of the topological group $G$ on the space $X$. For every $g \in G$, the function $x \mapsto a(g, x)$ is a homeomorphism of $X$. We use $g x$ as an abbreviation for $a(g, x)$. This notation is sometimes slightly confusing, especially if $G$ is a group of homeomorphisms on some space. The action is called transitive if for all $x, y \in X$ there exists $g \in G$ such that $g x=y$. This implies that $X$ is homogeneous. If $H$ is a closed subgroup of a topological group $G$, then $G$ acts transitively on the coset space $G / H=\{x H: x \in G\}$ and the natural projection map $\pi: G \rightarrow G / H$ is open. If $G$ acts on $X$, then the closed subgroup $G_{x}=\{g \in G: g x=x\}$ of $G$ is called the stabilizer of $x \in X$. It is well-known, and easy to prove, that if $G$ acts transitively, then $G / G_{x}$ is homeomorphic to $X$ 
if for every open neighborhood of the neutral element $e$ of $G$ and for some $x \in X$ (equivalently, for every $x \in X$ ) we have that $U x$ is open. If the action has this property, then it is called micro-transitive. So for a space $X$ to be (homeomorphic to) a coset space, it is necessary and sufficient that there is a topological group $G$ acting transitively and micro-transitively on $X$. The Effros Theorem from Effros [9] (see also [1, [12] and [20]) implies that if both $G$ and $X$ are Polish and the action is transitive, then it is micro-transitive. This result was generalized in van Mill [20]: it suffices to assume that $G$ is analytic and $X$ is of the second category.

For a space $X$ we let $\mathcal{H}(X)$ denote its group of homeomorphisms. If $X$ is compact, then the so-called compact-open topology on $\mathcal{H}(X)$ makes it a Polish group. A subbasis for the compact-open topology on $\mathcal{H}(X)$ consists of all sets of the form

$$
[K, U]=\{f \in \mathcal{H}(X): f(K) \subseteq U\},
$$

where $K$ and $U$ are arbitrary subsets of $X$ with $K$ compact and $U$ open. It is not difficult to prove that the function $(g, x) \mapsto g(x)$ is an action of $\mathcal{H}(X)$ on $X$.

Let $X$ and $(Y, \varrho)$ be spaces. If $f: A \rightarrow Y$ is continuous and $A \subseteq X$ is dense, then we say that the oscillation of $f$ at $x \in X$ is zero if for every $\varepsilon>0$ there exists a neighborhood $U$ of $x$ in $X$ such that the $\varrho$-diameter of $f(U \cap A)$ is less

than $\varepsilon$. Evidently, the set of all points in $X$ at which the oscillation of $f$ is zero is a $G_{\delta}$-subset of $X$ containing $A$. It is well-known, and easy to prove, that for complete $\varrho$ the function $f$ can be extended to a continuous function $\bar{f}: B \rightarrow Y$, where $B$ is the set of all points of $X$ at which the oscillation of $f$ is zero [10, 4.3.16].

As usual, we let $\mathfrak{c}$ denote the cardinality of the continuum.

\section{Spaces that Do OR DO NOT have the SP}

In this section we present several examples of spaces that do or do not have the SP.

(A) Properties of spaces with the SP. Our first results show that SP-spaces are close to being complete.

Proposition 3.1. Let $X$ be a space. Then

(1) If $X$ has the SP, then $X$ is Baire.

(2) If $X$ contains a dense Polish subspace and has the SP, then $X$ is completely Baire.

Proof. For (1), assume that $X$ is not Baire. Let $\mathcal{U}$ be the collection of all open subsets $U$ of $X$ that belong to $\operatorname{MGR}(X)$. Then $\mathcal{U} \neq \emptyset$, and there is a countable subcollection $\mathcal{V}$ of $\mathcal{U}$ such that $\bigcup \mathcal{V}=\bigcup \mathcal{U}$. This means that $A=\bigcup \mathcal{U} \in \operatorname{MGR}(X)$. Pick a point $x \in A$, and let $f: X \rightarrow X$ be a homeomorphism such that $f(x) \notin A$. There is an element $U \in \mathcal{U}$ containing $x$. But then $f(U)$ belongs to $\mathcal{U}$ since $f$ is a homeomorphism, and $f(U) \nsubseteq A$, which evidently is a contradiction.

We now prove (2). Let $G \subseteq X$ be a dense Polish subspace in $X$. If $X$ is not completely Baire, then it contains a closed subspace $A$ which is homeomorphic to the space of rational numbers $\mathbb{Q}$ (see $\$ 2$ ). Let $f: X \rightarrow X$ be a homeomorphism such that $(X \backslash G) \cap f(A)=\emptyset$. Then $f(A)$ is a closed copy of $\mathbb{Q}$ in the Polish space $G$, which is clearly impossible. 
Corollary 3.2. Let $X$ be a space.

(1) If $X$ is co-analytic, contains a dense Polish subspace and has the SP, then $X$ is Polish.

(2) If $X$ is analytic and has the SP, then $X$ has a dense Polish subspace and is completely Baire.

(3) If $X$ is Borel and has the SP, then $X$ is Polish.

Proof. First observe that (1) is a direct consequence of Proposition 3.1(2) and Lemma 2.3. For (2), first observe that $X$ is Baire by Proposition 3.1(1). Hence $X$ has a dense Polish subspace by Lemma 2.2. It consequently suffices to apply Proposition 3.1(2).

Finally, (3) is a direct consequence of (2) and (1).

Remark 3.3. In [13, Hrušák and Zamora Avilés proved that if $X$ is Borel and CDH, then $X$ is Polish. We pointed out in $₫ 1$ that a Baire $\mathrm{CDH}$-space has the SP. But since not every $\mathrm{CDH}$-space is Baire [11, Corollary [3.2(3) cannot be thought of as a strengthening of the just-quoted result of Hrušák and Zamora Avilés. But they are very strongly related of course, both in spirit and in their proofs.

Note that Corollary 3.2(3) is the statement of Theorem 1.1.

(B) Classes of spaces with the SP. We will now identify some classes of spaces that have the SP.

Proposition 3.4. Let $G$ be a Baire group acting transitively on a second category space $X$. Then for all subsets $A$ and $B$ of $X$ with $A$ countable and $B$ first category, the set of elements $g \in G$ such that $g A \cap B=\emptyset$ is dense in $G$.

Proof. We may assume without loss of generality that $B$ can be written as $\bigcup_{n<\omega} B_{n}$, with every $B_{n}$ closed and nowhere dense.

For $a \in A$ and $n<\omega$, put $G_{a, n}=\left\{g \in G: g a \notin B_{n}\right\}$. Then evidently, $G_{a, n}$ is open. We will prove that it is dense as well. To see this, take an arbitrary $h \in G$ with open neighborhood $U$ in $G$. Since $G$ is Lindelöf, there is a countable $F \subseteq G$ such that $F U=G$. Observe that since $G$ acts transitively,

$$
X=G a=(F U) a=F(U a)=\bigcup\{f(U a): f \in F\} .
$$

For every $f \in F$, the homeomorphism $x \mapsto f x$ of $X$ maps $U a$ onto $f(U a)$; hence $U a \in \operatorname{MGR}(X)$ if and only if $f(U a) \in \operatorname{MGR}(X)$. Since $X \notin \operatorname{MGR}(X)$ and $F$ is countable, this implies that $U a \notin \operatorname{MGR}(X)$. Hence $U a \backslash B_{n} \neq \emptyset$, so we may pick $h^{\prime} \in U$ such that $h^{\prime} a \notin B_{n}$.

Now since $G$ is Baire, the set $\bigcap_{a \in A} \bigcap_{n<\omega} G_{a, n}$ is dense in $G$. This clearly finishes the proof.

Remark 3.5. It is a natural question whether a Baire group pushes arbitrary first category subsets apart. But this is not true since there is a first category subset $A$ of $\mathbb{R}$ such that $(x+A) \cap A \neq \emptyset$ for every $x \in \mathbb{R}$. Indeed, let $\triangle$ denote the standard Cantor middle-third set in $\mathbb{I}$. It is well known that every $x \in[-1,1]$ can be written as $x=\alpha-\beta$, where $\alpha, \beta \in \triangle$; cf. [19, 1.5.9]. Using this, it is easy to see that $A=\mathbb{Z}+\triangle$ is as required.

Let $X$ be a homogeneous locally compact space, and consider its Alexandrov one-point compactification $\alpha X=X \cup\{\infty\}$. The group

$$
\{g \in \mathcal{H}(\alpha X): g(\infty)=\infty\}
$$


is closed in $\mathcal{H}(\alpha X)$, hence is Polish, and by homogeneity clearly acts transitively on $X$. Hence by Proposition 3.4 we consequently get our first important class of spaces with the SP:

Corollary 3.6. Every locally compact homogeneous space has the SP.

Remark 3.7. This means that the product of the Cantor set and the 1-sphere has the SP. Since a moment's reflection shows that it is not CDH, this shows that even in the class of compact topological groups the separation property is strictly weaker than the property of being countable dense homogeneous.

Remark 3.8. It is clear that the proof of Proposition 3.4 'works' if $G$ is Baire and $\aleph_{0}$-bounded, meaning that for every neighborhood $U$ of the identity of $G$ there is a countable subset $F$ of $G$ such that $F U=G$. This implies for example that if $X$ is a compact homogeneous Hausdorff space such that its group of homeomorphisms $\mathcal{H}(X)$ endowed with the compact-open topology is a Baire space and is $\aleph_{0}$-bounded, then $X$ has the SP. In Appendix 2 of this paper, we will present an example of a compact homogeneous Hausdorff space $X$ which does not have the SP. In fact, $\mathcal{H}(X)$ is neither a Baire space nor an $\aleph_{0}$-bounded topological group.

In view of Corollary 3.6, the question arises whether every homogeneous Polish space has the SP. The answer is in the negative, as the following example shows.

Example 3.9. There is a homogeneous Polish space $Z$ on which some Polish group $G$ acts such that

(1) $Z$ has precisely two $G$-orbits $A$ and $B$ such that $A$ is Polish and $B$ is meager. Moreover, $B$ has only countably many components.

(2) If $C$ is a component of $Z$ with $C \cap A \neq \emptyset$, then $C \subseteq A$, and similarly for $B$.

(3) For every point $z \in Z$ there is a sequence of components of $B$ that converges to $z$. In contrast, no sequence of components of $A$ converges to a point.

(4) No topological group acts transitively on $Z$.

(5) $Z$ does not have the SP.

Proof. This is example $Z$ in 22, where (1) through (4) are proved. We will check that (5) follows from (1) through (3). First observe that by (2), every component of $A$ is a component of $Z$, and similarly for $B$. Now let $D$ be a countable subset of $B$ which intersects every component of $B$ and, striving for a contradiction, assume that there is a homeomorphism $f: Z \rightarrow Z$ such that $f(D) \cap B=\emptyset$. A moment's reflection shows that $f(B) \subseteq A$. Since $f$ permutes the components of $Z$, it follows by the first part of (3) that $A$ contains a sequence of components converging to a point. But this contradicts the second part of (3).

Corollary 3.2(3) shows that Borel spaces with the SP are Polish. Hence spaces with the SP that are not Polish are rare. We will present such examples below. Our aim is now to formulate a simple and usable criterion that detects the SP in Polish spaces. Let $X$ be a space with open cover $\mathcal{U}$. We say that a map $f: X \rightarrow X$ is limited by $\mathcal{U}$ if for each $x \in X$ there is an element $U \in \mathcal{U}$ containing both $x$ and $f(x)$. It was shown in [2, Lemma 5.1] that if $X$ is Polish and $\left\{h_{n}\right\}_{n}$ is a sequence of homeomorphisms of $X$ for which there exists a sequence of open covers $\left\{\mathcal{U}_{n}\right\}_{n}$ of $X$ such that

(1) $\mathcal{U}_{n}$ is a barycentric refinement of $\mathcal{U}_{n-1}$;

(2) $\mathcal{U}_{n}$ has mesh less than $2^{-n}$; 
(3) $\left(h_{n} \circ \cdots \circ h_{0}\right)^{-1}\left(\mathcal{U}_{n}\right)$ has mesh less than $2^{-n}$;

(4) $h_{n}$ is limited by $\mathcal{U}_{n}$,

then $\lim _{n \rightarrow \infty} h_{n} \circ \cdots \circ h_{0}$ is a homeomorphism of $X$. (We use a complete metric on $X$ of course.) This is a form of the so-called Inductive Convergence Criterion for Polish spaces. This result leads us to the result we are after.

Theorem 3.10. Let $X$ be a Polish space with the following property: for every $x \in X$, each nowhere dense subset $A$ of $X$, and each open cover $\mathcal{U}$ of $X$, there exists a homeomorphism $f: X \rightarrow X$ that is limited by $\mathcal{U}$ while moreover $f(x) \notin A$. Then $X$ has the SP.

Proof. Let $D$ and $A$ be subsets of $X$ with $D$ countable and $A \in \operatorname{MGR}(X)$. We may assume that $A$ can be written as $\bigcup_{n<\omega} A_{n}$, with each $A_{n}$ closed and nowhere dense. We will construct a sequence of homeomorphisms $\left(f_{n}\right)_{n}$ of $X$ that satisfies the conditions mentioned in the Inductive Convergence Criterion such that $f=$ $\lim _{n \rightarrow \infty} f_{n} \circ \cdots \circ f_{0}$ is such that $f(D) \cap A=\emptyset$. The inductive construction of this sequence is completely trivial. To see this, pick an arbitrary point $d \in D$. The conditions imply that there is an 'arbitrarily close to the identity' homeomorphism $f_{0}: X \rightarrow X$ such that $f_{0}(d) \notin A_{0}$. But then $\varrho\left(f_{0}(d), A_{0}\right)>0$, and so we can easily arrange that in the limit $f_{0}(d)$ is not carried back to $A_{0}$. So in an infinite process we can free each point in $D$ from each of the $A_{n}$ 's. The details of checking this are left to the reader.

Corollary 3.11. Let $X$ be a Polish SLH-space. Then $X$ has the SP.

We will demonstrate that completeness is essential in this result.

Example 3.12. There is a connected Baire space that is SLH but does not have the SP.

Proof. The example $X$ in [17, 3.5] is a connected Baire space, is SLH, and has a countable dense subset $E$ such that $f(E) \cap E \neq \emptyset$ for any homeomorphism $f: X \rightarrow$ $X$. Hence $X$ does not have the SP.

The assumption on SLH-ness in Corollary 3.11 is essential as well, even among homogeneous spaces, as is demonstrated by Example 3.9.

(C) More examples. We will indicate now that the results in this section are more or less optimal. Recall that a subset $X$ of the Cantor set $2^{\omega}$ is $\aleph_{1}$-dense provided that every nonempty open subset of $2^{\omega}$ intersects $X$ in a set of size $\aleph_{1}$. The following result is Theorem 2.6 in Hrušák and Zamora Avilés [13].

Theorem $3.13\left(\mathrm{MA}+\neg \mathrm{CH}+\omega_{1}=\omega_{1}^{L}\right)$. Let $X$ be an $\aleph_{1}$-dense subset of $2^{\omega}$. Then

(1) $X$ is a co-analytic first category $\mathrm{CDH}$-space.

(2) $Y=2^{\omega} \backslash X$ is an analytic completely Baire $\mathrm{CDH}$-space which is not Polish.

Let $Y$ be as in Theorem 3.13(2). Observe that $Y$ has the SP(see \$1) and hence has a dense Polish subspace by Corollary 3.2(2) (this can also be seen directly of course). Hence $Y$ demonstrates that Corollary 3.2(3) unfortunately cannot be generalized to analytic spaces.

Observe that $Y$ is not even a Borel space. If it were, then $X$ would be Borel, contradicting the fact that an uncountable analytic space contains a Cantor set and hence has cardinality $\mathfrak{c}>\aleph_{1}$. 
Remark 3.14. One can think of Theorem 1.2 as a generalization of Theorem 1.1 beyond Borel spaces. Stronger generalizations will be difficult to establish. See the remarks about the Axiom of Projective Determinacy and the Axiom of Determinacy in Hrušák and Zamora Avilés [13].

\section{Proof of Theorem 1.2}

Let $G$ be an analytic group acting on a space $X$. Assume that for all subsets $A$ and $B$ of $X$ with $A$ countable and $B \in \operatorname{MGR}(X)$ there is an element $g \in G$ such that $g A \cap B=\emptyset$. We will prove that $X$ is Polish.

Lemma 4.1. Every orbit of $G$ is clopen in $X$ and contains a dense Polish subspace.

Proof. We will complete the proof of the lemma in several steps.

Claim 1. If $x \in X$, then $G x \notin \operatorname{MGR}(X)$ and is a Baire space.

Proof. If $G x \in \operatorname{MGR}(X)$, then there is by assumption an element $g \in G$ such that $g x \notin G x$ and this is nonsense. So every orbit of $G$ is second category in $X$ (and hence in itself). Since orbits are homogeneous, this implies that every orbit of $G$ is a Baire space.

Claim 2. If $x \in X$, then $G x$ is closed and contains a dense Polish subspace.

Proof. Since the map $g \mapsto g x$ is a continuous surjection $G \rightarrow G x$, it follows that $G x$ is analytic. By Claim 11 we conclude that $G x$ is a Baire space; hence it has by Lemma 2.2 a dense Polish subspace, say $S$.

Striving for a contradiction, assume that there is an element $p \in \overline{G x} \backslash G x$. Observe that $G p \subseteq \overline{G x} \backslash G x$. Hence $\overline{G x} \backslash S \in \operatorname{MGR}(X)$ and contains $G p$. So $G p \in \operatorname{MGR}(X)$ and we contradict Claim 1 .

Claim 3. If $x \in X$, then $G x$ is open.

Proof. Since $G x \notin \operatorname{MGR}(X)$ and is closed by Claims 1 and 2, it has nonempty interior. But then, clearly, $G x$ is open.

So we are done.

Hence the orbits of $G$ form a (necessarily countable) clopen partition of $X$. Fix an arbitrary $x \in X$, and consider its orbit $G x$. Assume that $A$ and $B$ are subsets of $G x$ with $A$ countable and $B \in \operatorname{MGR}(G x)$. Then $B \in \operatorname{MGR}(X)$. Hence there exists by assumption an element $g \in G$ such that $g A \cap B=\emptyset$. Clearly, $g A \subseteq G x$. Hence $G$ acts on $G x$ in the same way as it acts on $X$. As a consequence we may assume without loss of generality that $G$ acts transitively on $X$. Since $G$ is analytic, this implies that $X$ is analytic.

By Lemma 4.1, pick a dense Polish subspace $T$ in $X$. In addition, let $\tilde{X}$ be an arbitrary completion of $X$. Write $\tilde{X} \backslash T$ as $\bigcup_{n<\omega} A_{n}$, where every $A_{n}$ is closed in $\tilde{X}$. Observe that since $T$ is dense in $\tilde{X}, A_{n} \cap X \in \operatorname{MGR}(X)$ for every $n<\omega$.

Let $a: G \times X \rightarrow X$ denote the action of $G$ on $X$.

Lemma 4.2. There is a Borel set $B$ in $\tilde{X}$ containing $X$ such that a can be extended to a continuous function $\bar{a}: G \times B \rightarrow \tilde{X}$ such that $\bar{a}(G \times(B \backslash X)) \cap X=\emptyset$. 
Proof. We let $\pi: G \times \tilde{X} \rightarrow \tilde{X}$ denote the projection. By [19, A.8.4] there is a $G_{\delta^{-}}$ subset $S$ of $G \times \tilde{X}$ containing $G \times X$ such that $a$ can be extended to a continuous function $\bar{a}: S \rightarrow \tilde{X}$. Put $C=\tilde{X} \backslash \pi((G \times \tilde{X}) \backslash S)$. Observe that $(G \times \tilde{X}) \backslash S$ is analytic since $G$ is analytic. Hence $C$ is co-analytic. Moreover, $C$ contains $X$ and $G \times C \subseteq S$. Now by the separation theorem for analytic sets, [15, 14.7], there is a Borel set $B$ of $\tilde{X}$ such that $X \subseteq B \subseteq C$. We claim that it is as we want. Assume that there are $p \in B$ and $g \in G$ such that $\bar{a}(g, p)=x \in X$. Pick a sequence $\left(x_{n}\right)_{n}$ of elements of $X$ converging to $p$. Then by continuity of $\bar{a}$, the sequence $\left(g x_{n}\right)_{n}=\left(\bar{a}\left(g, x_{n}\right)\right)_{n}$ converges to $\bar{a}(g, p)=x$. Hence $g x_{n} \rightarrow x$, but then, since $G$ acts on $X, x_{n} \rightarrow g^{-1} x \in X$. Hence $p$ belongs to $X$ being equal to $g^{-1} x$.

Remark 4.3. Instead of Lemma 4.2, we could have used Theorem 2.2.7 in Becker and Kechris [4]. However, for the proof of our theorem the classical Lavrentieff Theorem suffices and so we decided not to use the more powerful Becker-Kechris result. Moreover, the proof of Theorem 2.2.7 in [4] contains a small inaccuracy which fortunately can easily be corrected. We will come back to this in Appendix 1 of this paper.

By abuse of notation, if $U \subseteq G$ and $p \in B$, we denote $\bar{a}(U \times\{p\})$ by $U p$. Moreover, if $U$ is a singleton, say $\{u\}$, then we denote $U p$ by $u p$.

Let $P$ be a Polish space for which there exists a continuous surjection $\alpha: P \rightarrow G$.

Let $\mathcal{S}$ be the collection of all subsets $S$ of $G$ for which there exists an element $x_{S} \in X$ such that $S x_{S} \in \operatorname{MGR}(X)$. Observe that $\emptyset \in \mathcal{S}$.

Let $\mathcal{U}$ be the collection of all open subsets of $P$ such that $\alpha(U)$ can be covered by countably many element of $\mathcal{S}$.

Lemma 4.4. $P \backslash \bigcup U \neq \emptyset$.

Proof. There is a countable subcollection $\mathcal{E}$ of $\mathcal{U}$ such that $\bigcup \mathcal{E}=\bigcup \mathcal{U}$. For every $U \in \mathcal{E}$ there is a collection $\left\{S_{n}^{U}: n<\omega\right\}$ of subsets of $G$ and for every $n$ an element $x_{n}^{U} \in X$ such that

(1) $S_{n}^{U} x_{n}^{U} \in \operatorname{MGR}(X)$

(2) $\alpha(U) \subseteq \bigcup_{n<\omega} S_{n}^{U}$.

Put $D=\left\{x_{n}^{U}: U \in \mathcal{E}, n<\omega\right\}$. There is an element $g \in G$ such that

$$
g D \cap \bigcup_{U \in \mathcal{E}} \bigcup_{n<\omega} S_{n}^{U} x_{n}^{U}=\emptyset .
$$

Then, clearly, $g \notin \bigcup_{U \in \mathcal{E}} \alpha(U)$.

So $P^{\prime}=P \backslash \bigcup U$ is a nonempty closed subset of $P$ and hence is Polish.

Corollary 4.5. If $V \subseteq P^{\prime}$ is a nonempty relatively open set, then $\alpha(V)$ cannot be covered by a countable subfamily of $\mathcal{S}$.

Proof. Suppose not. Pick a countable subfamily $\mathcal{E}$ of $\mathcal{U}$ such that $\bigcup \mathcal{E}=\bigcup \mathcal{U}$. It follows easily that $V \cup\left(P \backslash P^{\prime}\right)=V \cup \bigcup \mathcal{E}$ belongs to $\mathcal{U}$, which is impossible since $V$ is a nonempty subset of $P^{\prime}$.

Let $\mathcal{W}$ be a countable base for $P^{\prime}$. We assume without loss of generality that $\emptyset \notin \mathcal{W}$. For $W \in \mathcal{W}$ and $n<\omega$, put

$$
S(W)_{n}=\left\{b \in B \backslash X: \alpha(W) b \subseteq A_{n}\right\} .
$$


Lemma 4.6. If $W \in \mathcal{W}$ and $n<\omega$, then $\overline{S(W)_{n}} \cap X=\emptyset$.

Proof. Suppose that there is a sequence $\left(b_{m}\right)_{m}$ in $S(W)_{n}$ with limit $x \in X$. Since $A_{n}$ is closed, it clearly follows that $\alpha(W) x \subseteq A_{n}$; hence $\alpha(W) x \subseteq A_{n} \cap X \in$ $\operatorname{MGR}(X)$. So $\alpha(W) \in \mathcal{S}$, which contradicts Corollary 4.5.

Lemma 4.7. $B \backslash X \subseteq \bigcup_{W \in \mathcal{W}} \bigcup_{n<\omega} S(W)_{n}$.

Proof. Take $b \in B \backslash X$ arbitrarily. Observe that by Lemma 4.2, $\alpha\left(P^{\prime}\right) b \subseteq \tilde{X} \backslash X \subseteq$ $\bigcup_{n<\omega} A_{n}$. For every $n<\omega$, put

$$
P_{n}=\left\{p \in P^{\prime}: \alpha(p) b \in A_{n}\right\} .
$$

Then $P_{n}$ is closed in $P^{\prime}$, and $\bigcup_{n<\omega} P_{n}=P^{\prime}$. Hence by the Baire Category Theorem for $P^{\prime}$, there exist $n<\omega$ and $W \in \mathcal{W}$ such that $W \subseteq P_{n}$. Hence $\alpha(W) b \subseteq A_{n}$, and so $b \in S(W)_{n}$.

Hence Lemmas 4.6 and 4.7 show that $X$ is a Borel space. Since it is also an SPspace, it is Polish by Corollary [3.2(3). This completes the proof of Theorem 1.2 .

Remark 4.8. In view of Theorem 1.2, it is natural to ask the following. Suppose that $G$ is an analytic group acting on a space $X$. Assume that for every countable subset $A$ of $X$ there is an element $g \in G$ such that $g A \cap A=\emptyset$. Is $X$ Polish? The answer to this question is in the negative. For a counterexample, consider any uncountable first category topological group. Hence this shows that Theorem 1.2 is 'best possible'.

\section{Proof of Theorem 1.3}

Let $X$ and $Y$ be as in Theorem 3.13. We assume $\mathrm{MA}+\neg \mathrm{CH}+\omega_{1}=\omega_{1}^{L}$ in this section and hence that the conclusions of Theorem 3.13 hold.

(A) Pushing Theorem 1.2 further for $Y$. We will first show that the proof of Theorem 1.2 can be used to get new information on $Y$.

Theorem 5.1. If $G$ is an analytic group acting on $Y$, then there is a countable subset $D$ of $Y$ such that $g D \cap D \neq \emptyset$ for every $g \in G$.

Note that since $Y$ is not Polish, Theorem 1.2 gives us that there exist subsets $A$ and $B$ of $Y$ with $A$ countable and $B \in \operatorname{MGR}(Y)$ such that $g A \cap B \neq \emptyset$ for every $g \in G$. The proper generalization of this to countable sets would be to demand that both $A$ and $B$ are countable. It is easy to see that this is equivalent to the statement of Theorem 5.1 .

Proof. The proof is by contradiction, i.e., we assume that for every countable subset $D$ of $Y$ there is an element $g \in G$ with $g D \cap D=\emptyset$.

There is by Lemma 4.2 a Borel set $B$ in $2^{\omega}$ containing $Y$ such that the action $a: G \times Y \rightarrow Y$ can be extended to a continuous function $\bar{a}: G \times B \rightarrow 2^{\omega}$ such that $\bar{a}(G \times(B \backslash Y)) \cap Y=\emptyset$. Again by abuse of notation, if $U \subseteq G$ and $p \in B$, we denote $\bar{a}(U \times\{p\})$ by $U p$. Moreover, if $U$ is a singleton, say $\{u\}$, then we denote $U p$ by $u p$.

Let $P$ be a Polish space for which there exists a continuous surjection $\alpha: P \rightarrow G$. 
If $x \in Y$ and $h \in G$, then

$$
G_{x, h}=\{g \in G: g x=h x\} .
$$

Observe that $G_{x, h}$ is closed in $G$ and contains $h$.

Let $\mathcal{U}$ be the collection of all open subsets of $P$ for which there exist countable subsets $A \subseteq Y$ and $H \subseteq G$ such that

$$
\alpha(U) \subseteq \bigcup\left\{G_{x, h}: x \in A, h \in H\right\} .
$$

Claim 1. $P \backslash \bigcup U \neq \emptyset$.

Proof. There is a countable subcollection $\mathcal{E}$ of $\mathcal{U}$ such that $\bigcup \mathcal{E}=\bigcup \mathcal{U}$. So if $\mathcal{U}$ covers $P,\{\alpha(U): U \in \mathcal{E}\}$ covers $G$, and hence there are countable sets $A \subseteq Y$ and $H \subseteq G$ such that

$$
G=\bigcup\left\{G_{x, h}: x \in A, h \in H\right\} .
$$

Since $H$ is countable, there is a countable $D \subseteq Y$ such that $A \subseteq D$ and $h D=D$ for every $h \in H$. There is, by assumption, an element $g \in G$ such that $g D \cap D=\emptyset$. Then clearly $g \notin \bigcup\left\{G_{x, h}: x \in A, h \in H\right\}$, which is a contradiction.

So $P^{\prime}=P \backslash \bigcup U$ is a nonempty closed subset of $P$ and hence is Polish.

Claim 2. If $V \subseteq P^{\prime}$ is a nonempty relatively open set, then there do not exist countable subsets $A \subseteq Y$ and $H \subseteq G$ such that $\alpha(V) \subseteq \bigcup\left\{G_{x, h}: x \in A, h \in H\right\}$.

Proof. If this is not true, then pick a countable subfamily $\mathcal{E}$ of $\mathcal{U}$ such that $\bigcup \mathcal{E}=$ UU. It now easily follows that $V \cup\left(P \backslash P^{\prime}\right)=V \cup \bigcup \mathcal{E}$ belongs to $\mathcal{U}$, which is impossible since $V$ is a nonempty subset of $P^{\prime}$.

Let $\mathcal{W}$ be a countable open base for $P^{\prime}$. We assume without loss of generality that $\emptyset \notin \mathcal{W}$. For $W \in \mathcal{W}$, put

$$
S(W)=\{b \in B \backslash Y:|\alpha(W) b|=1\} .
$$

Claim 3. If $W \in \mathcal{W}$, then $\overline{S(W)} \cap Y=\emptyset$.

Proof. Suppose that there is a sequence $\left(b_{n}\right)_{n}$ in $S(W)$ with limit $y \in Y$. Pick an arbitrary $t \in W$, and put $c_{n}=\alpha(t) b_{n}$ and $c=\alpha(t) y$, respectively. Observe that $c_{n} \rightarrow c$. If $s \in W$ is arbitrary, then

$$
\alpha(s) y=\lim _{n \rightarrow \infty} \alpha(s) b_{n}=\lim _{n \rightarrow \infty} c_{n}=c .
$$

This proves that $\alpha(W) \subseteq G_{y, h}$, where $h=\alpha(t)$, and hence contradicts Claim 2 . $\diamond$

Now put $T=B \cap \bigcup_{W \in \mathcal{W}} \overline{S(W)}$. Then $T$ is an $F_{\sigma}$-subset of $B$ which misses $Y$ by Claim [3. Since $Y$ is not Borel, there is an element $b \in B \backslash(T \cup Y)$. Observe that the 'orbit' $G b$ of $b$ is contained in $2^{\omega} \backslash Y=X$.

Claim 4. $G b$ is uncountable.

Proof. Striving for a contradiction, assume that $G b$ is countable, say $G b=\left\{z_{n}\right.$ : $n<\omega\}$. For every $n$, put

$$
A_{n}=\left\{t \in P^{\prime}: \alpha(t) b=z_{n}\right\} .
$$

Then $A_{n}$ is clearly closed, and $\bigcup_{n<\omega} A_{n}=P^{\prime}$. By the Baire Category Theorem, the interior in $P^{\prime}$ of some $A_{n}$ is nonempty. But then there exists $W \in \mathcal{W}$ such that $|\alpha(W) b|=1$, i.e., $b \in T$. This is a contradiction. 
This completes the proof since $G b$ is an uncountable analytic space, hence contains a Cantor set. This is impossible since $|X|=\aleph_{1}$ and $\mathfrak{c}>\aleph_{1}$.

Hence we conclude that an analytic group acting on $Y$ is somehow 'concentrated' on a countable subset of $Y$. But do there exist analytic groups that act in a nontrivial way on $Y$ ? This question will now be considered.

(B) $Y$ is homogeneous. We will prove that $Y$ is a homogeneous space.

Let $X$ be a space and fix an arbitrary point $x \in X$. By $\tau(x)$ we denote the type of $x$, i.e.,

$$
\{y \in X:(\exists h \in \mathcal{H}(X))(h(x)=y)\} .
$$

Observe that types are always homogeneous spaces.

For a $\mathrm{CDH}$-space $X$, types are particularly nice. Bennett $[\underline{6}$, proved that all types $\tau(x)$ for $x \in X$ are clopen (see also [19, 1.6.7]). This gives us that every CDH-space is the topological sum of an at most countable family of homogeneous spaces.

Now consider the space $Y$ and pick an arbitrary $y \in Y$. Since $Y$ is $\mathrm{CDH}, \tau(y)$ is clopen in $Y$. Let $U$ be an open subset of $2^{\omega}$ such that $U \cap Y=\tau(y)$. There is a nonempty clopen subset $C$ of $2^{\omega}$ such that $C \subseteq U$. Put $Y^{\prime}=C \cap Y$ and $X^{\prime}=X \cap C$, respectively. Then $Y^{\prime}$ is homogeneous, being a clopen subspace of the zero-dimensional homogeneous space $\tau(y)$. Also, $X^{\prime}$ is an $\aleph_{1}$-dense subset of $C$. Since $C$ and $2^{\omega}$ are homeomorphic, $Y$ and $Y^{\prime}$ are homeomorphic by Baldwin and Beaudoin [3, Lemma 3.1]. Hence $Y$ is homogeneous.

(C) $Y$ is a coset space of an analytic group. Since $Y$ is zero-dimensional and homogeneous, it is SLH. By van Mill [21, Theorem 3.2] there is an analytic group acting transitively on $Y$. Since $Y$ is second category, this action is micro-transitive by the improved Effros Theorem (see \$2). Hence $Y$ is a coset space of some analytic group.

\section{Appendix 1. On the LaVRentiefF theOREM For Group aCtions}

The classical Lavrentieff Theorem [10,4.3.21] was generalized by Becker and Kechris as follows:

Theorem A1.1 ([4, 2.2.7]). Let $G$ be a Polish group, $X$ a Polish space and $A \subseteq X$. If $a: G \times A \rightarrow A$ is a continuous action of $G$ on $A$, there is a co-analytic set $B$, with $A \subseteq B \subseteq X$, and a continuous action $b: G \times B \rightarrow B$ such that $a=b \uparrow(G \times A)$. Moreover, if $A$ is analytic, we can find such a $B$ which is Borel. Finally, if $G$ is locally compact, $B$ can be taken to be $G_{\delta}$.

It is asked in 4, 2.2.8] whether the set $B$ in Theorem A1.1 can be taken to be $G_{\delta}$. This question was solved in the negative recently by Ding and Gao [7. They proved that every nonlocally compact Polish group can serve as a counterexample: for every nonlocally compact Polish group $G$ there are a Polish space $X$, a $\boldsymbol{\Pi}_{1}^{1}$ complete subset $A$ of $X$ and a nonextendable continuous action $a: G \times A \rightarrow A$.

The aim of this appendix is to point out that the proof of Theorem A1.1 by Becker and Kechris contains a small inaccuracy. Fortunately, it can easily be corrected. We will also provide a negative answer to Question 4.7 in Ding and Gao [7].

Let $\triangle$ be the standard Cantor middle-third set. Let $\{E, F, G\}$ be a clopen partition of $\triangle$ by nonempty sets. Pick an arbitrary point $x \in E$, and write $E \backslash\{x\}$ as the disjoint union of nonempty clopen subsets $\left\{E_{n}: n<\omega\right\}$. Similarly, pick $y \in F,\left\{F_{n}: n<\omega\right\}, z \in G$, and $\left\{G_{n}: n<\omega\right\}$. For $n<\omega$ let $f_{2 n}: E_{2 n} \rightarrow F_{n}$ 
and let $f_{2 n+1}: E_{2 n+1} \rightarrow G_{n}$ be arbitrary homeomorphisms. Let $f: E \backslash\{x\} \rightarrow$ $(F \backslash\{y\}) \cup(G \backslash\{z\})$ be the homeomorphism $\bigcup_{n<\omega} f_{n}$.

Put $A=\triangle \backslash\{x, y, z\}$, and define an involution $\sigma: A \rightarrow A$ by

$$
\sigma(u)= \begin{cases}f(u), & (u \in E \backslash\{x\}), \\ f^{-1}(u), & (u \in(F \backslash\{y\}) \cup(G \backslash\{z\})) .\end{cases}
$$

Lemma A1.2. The oscillation of $\sigma$ at $y$ and $z$ is zero and $\bar{\sigma}(y)=\bar{\sigma}(z)=x$, while the oscillation of $\sigma$ at $x$ is not zero.

Proof. Let $U$ be a neighborhood of $x$ which is contained in $E$. Then clearly $V(U)=$ $f(U \backslash\{x\}) \cup\{y, z\}$ is a neighborhood of $\{y, z\}$ which is contained in $F \cup G$.

We prove simultaneously that the oscillation of $\sigma$ at $y$ is zero and that $\bar{\sigma}(y)=$ $x$. Let $U$ have small diameter. Then $V(U)$ is a neighborhood of $y$ such that $\sigma(V(U) \cap A) \subseteq U$ and hence has small diameter, and similarly for $z$.

On the other hand, no matter how small we take $U$, it will always contain a tail of the sequence $\left(E_{n}\right)_{n}$. Hence $\sigma(U)$ contains tails of the sequences $\left(F_{n}\right)_{n}$ and $\left(G_{n}\right)_{n}$. This means that the diameter of $\sigma(U)$ is always larger than or equal to, say, the distance of $y$ and $z$ divided by 2 .

Let $G=\mathbb{Z}_{2}=\{\mathbf{0}, \mathbf{1}\}, A$ as above and $X=\triangle$. The involution $\sigma$ on $A$ defines in the obvious way an action $a: G \times A \rightarrow A$. Put

$$
B=\{s \in X:(\forall g \in G) \text { (the oscillation of } a \text { at }(g, s) \text { is zero) }\} .
$$

Observe that the oscillation of $a$ is zero at every point of the form $(\mathbf{0}, s)$, where $s \in X$. Hence $B$ is simply the set of all $s \in X$ at which the oscillation of $\sigma$ is zero. So we conclude from Lemma A1.2 that $B=\triangle \backslash\{x\}$. The action $a: G \times A \rightarrow A$ can however not be extended to an action $b: G \times B \rightarrow B$ since by Lemma A1.2 this would imply that $b(\mathbf{1}, y)=x \notin B$, contrary to what is asserted in the proof of 4 , 2.2.7].

Now that we know what the problem is, it is a triviality to fix it by a slight modification of the proof of [4, 2.2.7]. We remark that the proof works for analytic groups, not only for Polish groups.

Theorem A1.3. Let $G$ be an analytic group, $X$ a Polish space and $A \subseteq X$. If $a: G \times A \rightarrow A$ is a continuous action of $G$ on $A$, there is a co-analytic set $B$, with $A \subseteq B \subseteq X$, and a continuous action $b: G \times B \rightarrow B$ such that $a=b \uparrow(G \times A)$. Moreover, if $A$ is analytic, we can find such a $B$ which is Borel. Finally, if $G$ is locally compact, $B$ can be taken to be $G_{\delta}$.

Proof. We may assume that $A$ is dense in $X$. Let $\hat{G}$ be a Polish space containing $G$ as a dense subspace, and let $\pi: \hat{G} \times X \rightarrow X$ denote the projection. Put

$$
Z=\{(g, x): g \in \hat{G}, x \in X \text {, and the oscillation of } a \text { at }(g, x) \text { is zero }\} .
$$

Then $Z$ is a $G_{\delta}$-subset of the Polish space $Y=\hat{G} \times X$ containing $G \times A$, and the function $a: G \times A \rightarrow A$ can be extended to a continuous function $b: Z \rightarrow$ $X[10,4.3 .16]$. Observe that $G \times X$ is analytic; hence $(G \times X) \backslash Z$ is analytic (Proposition 2.1). Hence $\pi((G \times X) \backslash Z)$ is analytic and clearly misses $A$. Put

$$
B_{0}=X \backslash \pi((G \times X) \backslash Z),
$$

and observe that

$$
B_{0} \in \Pi_{1}^{1}(X), \quad A \subseteq B_{0}, \quad G \times B_{0} \subseteq Z
$$


Now $b^{-1}\left(B_{0}\right) \in \Pi_{1}^{1}(Z)$ by Proposition 2.1(3), and $b^{-1}\left(B_{0}\right)$ contains $G \times A$. Hence $(G \times X) \backslash b^{-1}\left(B_{0}\right)$ is analytic, and so is $\pi\left((G \times X) \backslash b^{-1}\left(B_{0}\right)\right)$. Put

$$
B_{1}=B_{0} \backslash \pi\left((G \times X) \backslash b^{-1}\left(B_{0}\right)\right),
$$

and observe that by Proposition 2.1(1),

$$
B_{1} \in \Pi_{1}^{\mathbf{1}}(X), \quad A \subseteq B_{1}, \quad G \times B_{1} \subseteq b^{-1}\left(B_{0}\right) .
$$

Proceeding in this way, we find that there is a sequence $\left\{B_{n}: n<\omega\right\}$ of subsets of $X$ such that for every $n<\omega$,

$$
B_{n} \in \Pi_{1}^{1}(X), \quad A \subseteq B_{n+1} \subseteq B_{n}, \quad G \times B_{n+1} \subseteq b^{-1}\left(B_{n}\right) .
$$

Now put $B=\bigcap_{n<\omega} B_{n}$. Then $B \in \Pi_{1}^{1}(X)$ by Proposition 2.1(1), $A \subseteq B$, and by $(3)$,

$$
G \times B \subseteq b^{-1}(B) .
$$

Now the reasoning in $4,2.2 .7]$ can be followed verbatim to complete the proof.

As we stated at the beginning of this appendix, Ding and Gao 7 proved that for every nonlocally compact Polish group $G$ there are a Polish space $X$, a $\Pi_{1}^{1}$ complete subset $A$ of $X$ and a nonextendable continuous action $a: G \times A \rightarrow A$. Here nonextendable means that $A$ is not a proper subset of any subset $B$ of $X$ such that $a$ can be extended to a continuous action $b: G \times B \rightarrow B$. They asked the following:

Question A1.4 (Ding and Gao [7, 4.7]). If $G$ is a Polish group acting on a subset $A$ of a Polish space $X$ and the action cannot be extended, is there a Polish $G$ space $X^{\prime}$ and a homeomorphic embedding of $X$ into $X^{\prime}$ whose restriction on $A$ is a $G$-embedding?

Informally, can the fact that the action is not extendable be remedied by expanding the space? We will show that this is not always possible. We will show that $G$ and $A$ as described earlier solve Question A1.4 in the negative. Indeed, suppose that there is a Polish space $X$ containing $\triangle$ such that the action $G \times A \rightarrow A$ can be extended to an action $G \times X \rightarrow X$. Since $A$ is $G$-invariant, so is its closure $\triangle$. This is a contradiction.

\section{Appendix 2. A homogeneous compact Hausdorff space}

In this appendix all spaces under discussion are Tychonoff. Let $\mathbb{S}$ denote the Sorgenfrey line.

Our aim is to describe an example of a homogeneous compact Hausdorff space $Z$ which does not have the SP. In fact, $Z$ contains a countable dense subset $E$ such that $h(E) \cap E \neq \emptyset$ for every $h \in \mathcal{H}(Z)$. We will show that $\mathcal{H}(Z)$ endowed with the compact-open topology is not a Baire space and is not $\aleph_{0}$-bounded. So, as to be expected, familiar techniques in the metrizable context of producing new homeomorphisms from old ones by taking limits do not work in the general setting of compact Hausdorff spaces. See [5] for a similar result.

We assume the reader is familiar with the papers [17, 18. As was noted in [17, Remark 4.1], the construction presented there works for any $\mathbb{R}^{n}$, where $n \geq 1$.

Let $\mathcal{F}=\left\{f: \operatorname{dom}(f)\right.$ and range $(f)$ are $G_{\delta}$-subsets of $\mathbb{R}$ and $f: \operatorname{dom}(f) \rightarrow$ range $(f)$ is a homeomorphism $\}$. For every $x \in \mathbb{R}$ put $V(x)=x+\mathbb{Q}$. Let $X$ be the space constructed on [17, page 145] (with $\mathbb{R}$ taking the role of $\mathbb{R}^{2}$ ). We assume 
without loss of generality that $0 \notin X$, i.e., that $\mathbb{Q} \subseteq Y=\mathbb{R} \backslash X$. The crucial property of $X$ is

Fact 1. If $f \in \mathcal{F}$ and $|\{x \in \operatorname{dom}(f): f(x) \notin V(x)\}|=\mathfrak{c}$, then there exists $x \in$ $\operatorname{dom}(f) \cap X$ such that $f(x) \notin X$.

Observe that $Y+\mathbb{Q}=Y$. We split each $y \in Y$ into two points, $y^{-}$and $y^{+}$. The points of $X$ will not be split. Order the set

$$
S(X)=X \cup\left\{y^{-}, y^{+}: y \in Y\right\}
$$

in the natural way, so that $y^{-}$always precedes $y^{+}$. Endow $S(X)$ with the order topology derived from this order. Then $S(X)$ is easily seen to be a locally compact, zero-dimensional first countable space. In addition, $X$ as a subspace of $\mathbb{R}$ is precisely the same space as $X$ as a subspace of $S(X)$. Moreover, the sets

$$
\left\{y^{+}: y \in Y\right\}, \quad\left\{y^{-}: y \in Y\right\}
$$

are both homeomorphic to subspaces of $\mathbb{S}$. The construction of splitting some but not all points of $\mathbb{R}$ is well-known and was used by many authors. See, e.g., van Douwen [8] and [18, $\S 6]$.

The following result is [18, Theorem 6.3].

Fact 2. $S(X)$ is homogeneous.

Now consider the clopen $\operatorname{arc} Z=\left[0^{+}, 1^{-}\right]$in $S(X)$. Then $Z$ is clearly compact and also homogeneous, being a clopen subspace of the homogeneous zerodimensional space $S(X)$ (Fact 2).

Let $D$ be a countable dense subset of $X$, and put $E=(D+\mathbb{Q}) \cap X^{\prime}$.

Let $f: Z \rightarrow Z$ be an arbitrary homeomorphism. We will show that $f(E) \cap E \neq \emptyset$.

Lemma A2.1. There is a countable subset $A$ of $X^{\prime}=X \cap(0,1)$ such that for $S=X^{\prime} \backslash A$ we have $f(S)=S$.

Proof. For every $n \in \mathbb{Z}$, put $A_{n}=\left\{x \in X^{\prime}: f^{n}(x) \notin X^{\prime}\right\}$. The function $d \mapsto f^{n}(d)$ embeds $A_{n}$ into $Z \backslash X^{\prime}$. Since $A_{n}$ has a countable base and $Z \backslash X^{\prime}$ is the union of two subspaces each homeomorphic to a subspace of $\mathbb{S}$, it follows that $A_{n}$ is countable (this is well-known; see, e.g., [18, 3.10]). Hence $A=\bigcup_{n \in \mathbb{Z}} A_{n}$ is countable. It is clear that $S=X^{\prime} \backslash A$ is as required.

Lemma A2.2. $f(E) \cap E \neq \emptyset$.

Proof. We adopt the notation in Lemma A2.1.

By [19, A.8.5], there are $G_{\delta}$-subsets $G$ and $H$ of $\mathbb{R} \backslash A$ such that $g=(f\lceil S): S \rightarrow S$ can be extended to a homeomorphism $\bar{g}: G \rightarrow H$. Hence $\bar{g} \in \mathcal{F}$, and if $\mid\{x \in G$ : $\bar{g}(x) \notin V(x) \mid=\mathfrak{c}$, then by Fact 1 there exists $x \in G \cap X$ such that $\bar{g}(x) \notin X$. But then $x \in S$ and $f(x)=\bar{g}(x) \notin X$, which contradicts Lemma A2.1. Hence we conclude that

$$
|\{x \in S: f(x) \notin V(x)\}|<\mathfrak{c} .
$$

For every $q \in \mathbb{Q}$, let $B_{q}=\left\{x \in X^{\prime}: f(x)=x+q\right\}$. We claim that $B_{q}$ is closed in $X^{\prime}$. To see this, let $\left(x_{n}\right)_{n}$ be a sequence in $B_{q}$ converging to an element $x \in X^{\prime}$. Then $f\left(x_{n}\right)$ converges on the one hand to $f(x) \in Z$ and on the other hand to $x+q \in X$. From this we conclude that $f(x)=x+q \in X^{\prime}$. 
From (1) and the fact that $A$ is countable, we conclude that

$$
\left|X^{\prime} \backslash \bigcup_{q \in \mathbb{Q}} B_{q}\right|<\mathfrak{c} .
$$

Hence Lemma 3.3 in [17] shows that there exists $q \in \mathbb{Q}$ such that $B_{q}$ has nonempty interior in $X^{\prime}$. But then $B_{q}$ meets $E$, say in $x$, and hence $f(x)=x+q \in E$.

Hence we conclude from Lemma A2.2 that $Z$ does not have the SP. We will now show that $\mathcal{H}(Z)$ is not a Baire space and is not $\aleph_{0}$-bounded.

Proposition A2.3. $\mathcal{H}(Z)$ is neither a Baire space nor an $\aleph_{0}$-bounded topological group.

Proof. Enumerate $E$ as $\left\{e_{n}: n<\omega\right\}$. For all $n, m<\omega$, put

$$
\mathcal{D}_{n, m}=\left\{f \in \mathcal{H}(Z): f\left(e_{n}\right)=e_{m}\right\} .
$$

Then by Lemma A2.2, $\mathcal{H}(Z)=\bigcup_{n, m<\omega} \mathcal{D}_{n, m}$. Hence it suffices to prove that for fixed $n, m<\omega$ we have that $\mathcal{D}_{n, m}$ is nowhere dense in $\mathcal{H}(Z)$. But this is a triviality. Indeed, pick a finite collection $\mathcal{K}$ of compact subsets of $Z$ and for every $K \in \mathcal{K}$ an open subset $U(K)$ in $Z$ such that $\bigcap_{K \in \mathcal{K}}[K, U(K)] \neq \emptyset$, say contains the homeomorphism $f$. We may assume without loss of generality that every $U(K)$ is clopen. If $f\left(e_{n}\right) \neq e_{m}$, then there is nothing to prove. Suppose therefore that $f\left(e_{n}\right)=e_{m}$. There is a clopen neighborhood $V$ of $e_{n}$ such that if $e_{m} \in U(K)$ for some $K \in \mathcal{K}$, then $f(V) \subseteq U(K)$, and if $e_{m} \notin U(K)$, then $f(V) \cap U(K)=\emptyset$. Since $f(V)$ is homogeneous, being clopen, there is a homeomorphism $\xi$ of $Z$ that is supported on $f(V)$ while moreover $\xi\left(e_{m}\right) \neq e_{m}$. Consider $g=\xi \circ f$. Then $g\left(e_{n}\right)=\xi\left(e_{m}\right) \neq e_{m}$; hence $g \notin \mathcal{D}_{n, m}$. It is easy to see that $g \in \bigcap_{K \in \mathcal{K}}[K, U(K)]$.

We next prove that $\mathcal{H}(Z)$ is not $\aleph_{0}$-bounded, as follows. We need the following fact, which is Lemma 6.2 from [18]: Let $\left[a^{+}, b^{-}\right]$and $\left[c^{+}, d^{-}\right]$be clopen $\operatorname{arcs}$ in $S(X)$ such that $c-a=d-b \in \mathbb{Q}$. Then $\left[a^{+}, b^{-}\right]$and $\left[c^{+}, d^{-}\right]$are order-isomorphic. Now for every $y \in Y \cap\left(0, \frac{1}{4}\right)$, consider the clopen arcs

$$
L_{y}=\left[y^{+},\left(y+\frac{1}{4}\right)^{-}\right], \quad R_{y}=\left[\left(y+\frac{1}{2}\right)^{+},\left(y+\frac{3}{4}\right)^{-}\right] .
$$

These arcs are order-isomorphic, hence homeomorphic, by what we just said. Since they are clopen and disjoint, there consequently is an involution $f_{y}$ of $Z$ which is supported on $L_{y} \cup R_{y}$ while moreover $f_{y}\left(L_{y}\right)=R_{y}$. Observe that for every $y \in Y \cap\left(0, \frac{1}{4}\right)$ we have

$$
f_{y}\left(\left[0^{+}, \frac{1}{2}^{-}\right]\right)=\left(\left[0^{+}, \frac{1}{2}^{-}\right] \backslash L_{y}\right) \cup R_{y} ;
$$

hence the collection

$$
\left\{f_{y}\left(\left[0^{+}, \frac{1}{2}^{-}\right]\right): y \in Y \cap\left(0, \frac{1}{4}\right)\right\}
$$

is uncountable.

Consider the open neighborhood

$$
\mathcal{P}=\left[\left[0^{+}, \frac{1}{2}^{-}\right],\left[0^{+}, \frac{1}{2}^{-}\right]\right] \cap\left[\left[\frac{1}{2}^{+}, 1^{-}\right],\left[\frac{1}{2}^{+}, 1^{-}\right]\right]
$$

of the identity homeomorphism of $Z$ (our notation is confusing since we use brackets with two different meanings), and let $F \subseteq \mathcal{H}(Z)$ be an arbitrary countable set. If 
$f \in F$, then $g \in f \mathcal{P}$ if and only if $g\left(\left[0^{+}, \frac{1}{2}^{-}\right]\right)=f\left(\left[0^{+}, \frac{1}{2}^{-}\right]\right)$. This means that if $g \in F \mathcal{P}$, then $g\left(\left[0^{+}, \frac{1}{2}^{-}\right]\right)$belongs to the countable collection

$$
\left\{f\left(\left[0^{+}, \frac{1}{2}^{-}\right]\right): f \in F\right\} .
$$

Hence by (3), there exists $y \in Y \cap\left(0, \frac{1}{4}\right)$ such that $f_{y} \notin F \mathcal{P}$.

This suggests the following questions.

Question A2.4. What are the compact (homogeneous) Hausdorff spaces $X$ for which $\mathcal{H}(X)$ is a Baire space?

Question A2.5. What are the compact (homogeneous) Hausdorff spaces $X$ for which $\mathcal{H}(X)$ is $\aleph_{0}$-bounded?

Remark A2.6. If $X$ is a homogeneous compact Hausdorff space for which $\mathcal{H}(X)$ is $\aleph_{0}$-bounded, then $X$ is Dugundji and hence dyadic. This follows from Uspenskiu [23, Theorem 1]. Since first countable or orderable dyadic compacta are metrizable (see [10, p. 231]), the fact that $\mathcal{H}(Z)$ is not $\aleph_{0}$-bounded is a consequence of Uspenskil's result. I am indebted to Shura Arhangel'skiı for drawing my attention to Uspenskiı's paper.

\section{REFERENCES}

[1] F. D. Ancel, An alternative proof and applications of a theorem of E. G. Effros, Michigan Math. J. 34 (1987), 39-55. MR873018 (88h:54058)

[2] R. D. Anderson, D. W. Curtis, and J. van Mill, A fake topological Hilbert space, Trans. Amer. Math. Soc. 272 (1982), 311-321. MR656491 (83j:57009)

[3] S. Baldwin and R. E. Beaudoin, Countable dense homogeneous spaces under Martin's axiom, Israel J. Math. 65 (1989), 153-164. MR998668 (90f:54010)

[4] H. Becker and A. S. Kechris, The descriptive set theory of Polish group actions, London Mathematical Society Lecture Note Series, vol. 232, Cambridge University Press, Cambridge, 1996. MR1425877 (98d:54068)

[5] D. P. Bellamy and K. F. Porter, A homogeneous continuum that is non-Effros, Proc. Amer. Math. Soc. 113 (1991), 593-598. MR:1070510 (91m:54016)

[6] R. Bennett, Countable dense homogeneous spaces, Fund. Math. 74 (1972), 189-194. MR0301711 (46:866)

[7] L. Ding and S. Gao, On generalizations of Lavrentieff's theorem for Polish group actions, Trans. Amer. Math. Soc. 359 (2007), 417-426 (electronic). MR 2247897 (2007e:54045)

[8] E. K. van Douwen, A compact space with a measure that knows which sets are homeomorphic, Adv. Math. 52 (1984), 1-33. MR.742164 (85k:28018)

[9] E. G. Effros, Transformation groups and $C^{*}$-algebras, Annals of Math. 81 (1965), 38-55. MR0174987 (30:5175)

[10] R. Engelking, General topology, Heldermann Verlag, Berlin, second ed., 1989. MR1039321 (91c:54001)

[11] I. Farah, M. Hrušák, and C. Martínez Ranero, A countable dense homogeneous set of reals of size $\aleph_{1}$, Fund. Math. 186 (2005), 71-77. MR2163103 (2006f:54031)

[12] A. Hohti, Another alternative proof of Effros' theorem, Top. Proc. 12 (1987), 295-298. MR991756 (90e:54090)

[13] M. Hrušák and B. Zamora Avilés, Countable dense homogeneity of definable spaces, Proc. Amer. Math. Soc. 133 (2005), 3429-3435. MR2161169(2006h:54026)

[14] W. Hurewicz, Relativ perfekte Teile von Punktmengen und Mengen (A), Fund. Math. 12 (1928), 78-109.

[15] A. S. Kechris, Classical descriptive set theory, Springer-Verlag, New York, 1995. MR1321597 (96e:03057)

[16] S. Levi, On Baire cosmic spaces, Proceedings of the Fifth Prague Topological Symposium, Heldermann Verlag, Praha, pp. 450-454. MR698438 (84d:54052) 
[17] J. van Mill, Strong local homogeneity does not imply countable dense homogeneity, Proc. Amer. Math. Soc. 84 (1982), 143-148. MR633296 (83e:54033)

[18] J. van Mill, Sierpiński's Technique and subsets of $\mathbb{R}$, Top. Appl. 44 (1992), 241-261. MR.1173263 (94d:54096)

[19] J. van Mill, The infinite-dimensional topology of function spaces, North-Holland Publishing Co., Amsterdam, 2001. MR.1851014 (2002h:57031)

[20] J. van Mill, A note on the Effros Theorem, Amer. Math. Monthly. 111 (2004), 801-806. MR2104051 (2005g:54069)

[21] J. van Mill, Homogeneous spaces and transitive actions by analytic groups, Bull. London Math. Soc. 39 (2007), 329-336. MR 2323467 (2008d:54031)

[22] J. van Mill, Homogeneous spaces and transitive actions by Polish groups, Israel J. Math. 165 (2008), 133-159. MR2403618

[23] V. V. Uspenskiǔ, Why compact groups are dyadic, General topology and its relations to modern analysis and algebra, VI (Prague, 1986), Res. Exp. Math., vol. 16, Heldermann, Berlin, 1988, pp. 601-610. MR952642 (89i:22005)

Faculty of Sciences, Department of Mathematics, VU University Amsterdam, De Boelelaan $1081^{a}, 1081$ HV Amsterdam, The Netherlands

E-mail address: vanmill@few.vu.nl 